\title{
Supervised exercise following bariatric surgery in morbid obese adults: CERT- based exercise study protocol of the EFIBAR randomised controlled trial
}

Emilio Villa-González ${ }^{1 *}$ D, Yaira Barranco-Ruiz ${ }^{1}$, Manuel A. Rodríguez-Pérez ${ }^{2,3}$, Alejandro Carretero-Ruiz ${ }^{2,3}$, José María García-Martínez ${ }^{2,3}$, Alba Hernández-Martínez ${ }^{2,3}$, María José Torrente-Sánchez ${ }^{4}$, Manuel Ferrer-Márquez ${ }^{4,5}$, Alberto Soriano-Maldonado ${ }^{2,3}$, Enrique G. Artero ${ }^{2,3}$ and On behalf of the EFIBAR Study Group

\begin{abstract}
Background: There is increasing evidence of weight regain in patients after bariatric surgery (BS), generally occurring from 12 to 24 months postoperatively. Postoperative exercise has been suggested to ad long-term weight maintenance and to improve physical function in BS patients. However, there are a limited number of intervention studies investigating the possible benefits of exercise in this population. The aim of the current report is to provide a comprehensive CERT (Consensus on Exercise Reporting Template)-based description of the rationale and details of the exercise programme implemented in the EFIBAR Study (Ejercicio FÍsico tras cirugía BARiátrica), a randomised controlled trial investigating the effects of a 16-week supervised concurrent (aerobic and strength) exercise intervention program on weight loss (primary outcome), body composition, cardiometabolic risk, physical fitness, physical activity and quality of life (secondary outcomes) in patients with severe/morbid obesity following bariatric surgery.
\end{abstract}

Methods: A total of 80 BS patients [60-80\% expected women, aged 18 to 60 years, body mass index $(B M I) \geq 40 \mathrm{~kg} / \mathrm{m}^{2}$ or $\geq 35 \mathrm{~kg} / \mathrm{m}^{2}$ with comorbid conditions)] will be enrolled in the EFIBAR Randomized Control Trial $(\mathrm{RCT})$. Participants allocated in the exercise group $(n=40)$ will undertake a 16-week supervised concurrent (strength and aerobic) exercise programme (three sessions/week, $60 \mathrm{~min} /$ session), starting 7 to 14 days after surgery. The rationale of the exercise programme will be described following the CERT criteria detailing the 16 key items. The study has been reviewed and approved by the Ethics Committee of the Torrecárdenas University Hospital (Almería, Spain) (ref. № 76/2016).

Discussion: The present study details the exercise programme of the EFIBAR RCT, which may serve: 1) exercise professionals who would like to implement an evidence-based exercise programme for BS patients, and 2) as an example of the application of the CERT criteria.

Trial registration: The trial was prospectively registered at Clinicaltrials.gov NCT03497546 on April 13, 2018. Keywords: Obese, Exercise programmes, Bariatric surgery, Adult and weight regain

\footnotetext{
*Correspondence: evilla@ugr.es

'Department of Physical and Sports Education, PROFITH "PROmoting FITness

and Health through Physical Activity" Research Group, Sport and Health

University Research Institute (iMUDS), Faculty of Education and Sport

Sciences, University of Granada, 52005 Melilla, Spain

Full list of author information is available at the end of the article
}

(c) The Author(s). 2019 Open Access This article is distributed under the terms of the Creative Commons Attribution 4.0 International License (http://creativecommons.org/licenses/by/4.0/), which permits unrestricted use, distribution, and

reproduction in any medium, provided you give appropriate credit to the original author(s) and the source, provide a link to the Creative Commons license, and indicate if changes were made. The Creative Commons Public Domain Dedication waiver (http://creativecommons.org/publicdomain/zero/1.0/) applies to the data made available in this article, unless otherwise stated. 


\section{Background}

Bariatric surgery (BS) is an effective weight loss intervention for morbidly obese patients, being successful in the treatment of obesity (stage I and II) and different associated diseases [1]. Nevertheless, there is increasing evidence of weight regain in patients after BS, $[2,3]$ usually occurring between 12 and 24 months postoperatively $[4,5]$. Weight regain increases the risk of physical function decline, which negatively affects the functionality of an individual to carry out tasks of daily life [5]. Weight regain also increases the likelihood of obesity-related comorbidity relapse [6], which makes it necessary to implement strategies to improve the lifestyle before [7] and after BS [8].

Postoperative lifestyle interventions combining diet, exercise and behaviour modification have proven successful in aiding long-term weight maintenance and improving physical function in BS patients [9]. However, there are a limited number of intervention studies investigating the possible multidimensional benefits of exercise in this population. For patients who have undergone BS and experience suboptimum weight loss, exercise could be an important adjunct therapy $[10,11]$. A previous review found a positive effect of exercise on anthropometric measurements, cardiovascular risk factors and physical fitness in BS patients [12], although results were not consistent, with a wide range of exercise programmes and perioperative timing, therefore hampering adequate practical guidance. Despite this heterogeneity, authors concluded that a beneficial exercise programme should last for a median of 12 weeks, have a mean intensity of $65 \%$ peak heart rate $/ \mathrm{VO}_{2 \max }$, and be at least partially supervised [12]. A recent metha-analysis concluded that exercise training programs performed after BS were effective to optimize weight loss and fat mass loss and to improve physical fitness, although no additional effect on lean body mass loss was found [13].

In particular, strength training increases muscular strength and attenuates muscle atrophy in obese adults adhering to caloric restriction for weight loss [14, 15], whereas aerobic training provides several metabolic improvements, such as attenuating the appearance of arterial stiffness [16]. Unfortunately, it is difficult to assess the appropriate type and amount of exercise in BS patients, as it is a relatively understudied population and a variety of training regimes have been described. To date, published evidence supports a potential role for exercise to elicit positive changes in body composition after BS. However, properly designed exercise-based randomised controlled trials (RCT) are needed to provide further evidence of the effectiveness of exercise as a feasible adjunct therapy to BS [17].

In general, descriptions of exercise intervention programmes in clinical research have traditionally been vague, often containing insufficient information to allow for replication. To address this, The Consensus on Exercise Reporting Template (CERT) was recently developed to standardise the reporting of exercise intervention programmes regardless of the population [18] CERT recommends that exercise programmes published in the literature should ideally report all of the components considered in the intervention, since these are essential to evaluate the effects of clinical trials, and the interpretation, translation and implementation of research findings into clinical practice. The Consensus provides guidance on a minimum set of 16 key items required to report replicable exercise programmes. While its development was stimulated by a metaepidemiological review of exercise interventions for chronic health conditions [19] it is equally applicable to describe exercise interventions for acute conditions, injury prevention or general health. Additionally, a recent report highlighted the importance of describing exercise programs following CERT recommendations, to facilitate the replicability of these programs in BS population [20].

The aim of the current report is to provide a comprehensive CERT-based description of the rationale and details of the exercise programme implemented in the EFIBAR (Ejercicio FÍsico tras cirugía BARiátrica) study, a 1-year RCT investigating the effects of a 16-week supervised concurrent (aerobic and strength) exercise intervention program on weight loss (primary outcome), body composition, cardiometabolic risk, physical fitness, physical activity and quality of life (secondary outcomes) in patients with severe/morbid obesity following bariatric surgery.

\section{Methods/design \\ Study design}

Briefly, a total of 80 BS patients [60-80\% expected women, aged 18 to 60 years, body mass index (BMI) $\geq 40$ $\mathrm{kg} / \mathrm{m}^{2}$ or $\geq 35 \mathrm{~kg} / \mathrm{m}^{2}$ with comorbid conditions)] will be enrolled in the EFIBAR Randomized Control Trial (RCT) (ClinicalTrials.gov ID: NCT03497546), following recruitment from a public hospital and a private clinic in Almería, southern Spain. Participants will be randomised either to a normal care Control Group $(n=40)$, involving nutritional status monitoring and diet/physical activity counselling following international guidelines [21], or an Exercise Group $(n=40)$, who will additionally undertake a 16-week supervised concurrent (strength and aerobic) exercise programme (three sessions/week, $60 \mathrm{~min} / \mathrm{session}$ ) starting 7 to 14 days after surgery, with the final aim of evaluating the effects on weight loss (primary outcome), body composition, cardiometabolic risk, physical fitness and quality of life (secondary outcomes). Recruitment of participants started in May 2018 and it may extend till December 2020.

Sample size calculations indicate that, assuming an alpha error of 0.05 and a power of $80 \%$, a total of 66 patients ( $n=33$ patients per group) will be needed to detect 
an effect (between group difference) of at least 0.7 standard deviations [22] in the main outcome (\% total weight loss, \%TWL). Anticipating a potential lost to follow-up of up to $20 \%$, a total of 80 patients will be recruited. Additionally, we will aim at maximizing the adherence and minimizing lost to follow-up, which could in fact increase the power to detect the main effect.

The rationale of the exercise programme implemented in EFIBAR will be described following the CERT criteria recommendations for detailing the 16 items (Table 1). The structure that was followed to describe the exercise programme is presented in the Fig. 1.

\section{Programme structure}

\section{Exercise programme rationale}

With the overall aim of making this exercise programme transferable to society, the exercise level in EFIBAR is based on the physical activity recommendations for adults proposed by the World Health Organization [23], together with the American College of Sports Medicine's position stand [24] on progression models in strength and aerobic training for healthy adults. Since there is limited information regarding the ideal exercise model for morbidly obese adults, we will combine strength and aerobic training (i.e., a concurrent training protocol), as previous findings in obese adults displayed important benefits when both strength and aerobic exercise are implemented in the same session [25]. A question that often arises is whether aerobic or strength training should be performed first within any particular training session. It has been suggested that performing aerobic exercise prior to strength training could result in impaired strength training performance (e.g., strength exercises technique), and unfavourable responses to strength (decrease in force production) when strength training is conducted with moderate to high loads in the general population [26]. Moreover, in a previous study conducted in obese men, concurrent strength prior to

Table 1 CERT checklist from EFIBAR study exercise programme

\begin{tabular}{|c|c|c|c|}
\hline & Item & Checklist Item & Identification (section) \\
\hline WHAT: materials & 1 & Detailed description of the type of exercise equipment & Additional file 1: Table S1 \\
\hline WHO: provider & 2 & Detailed description of the qualifications, expertise and/or training & Exercise programme characteristics \\
\hline \multirow[t]{10}{*}{ HOW: delivery } & 3 & $\begin{array}{l}\text { Describe whether exercises are performed individually or in a } \\
\text { group }\end{array}$ & Exercise programme characteristics \\
\hline & 4 & $\begin{array}{l}\text { Describe whether exercises are supervised or unsupervised; how } \\
\text { they are delivered }\end{array}$ & Exercise programme characteristics \\
\hline & 5 & $\begin{array}{l}\text { Detailed description of how adherence to exercise is measured } \\
\text { and reported }\end{array}$ & $\begin{array}{l}\text { Programme adherence } \\
\text { Tally sheet/daily control (Additional file 3: Table S3) }\end{array}$ \\
\hline & 6 & Detailed description of motivation strategies & $\begin{array}{l}16 \text { reinforcement WhatsApp messages / every } \\
\text { Friday (Additional file } 3 \text { ) } \\
4 \text { reinforcement videos / end of every month } \\
\text { (Additional file 3) }\end{array}$ \\
\hline & $7 a$ & $\begin{array}{l}\text { Detailed description of the decision rule(s) for determining } \\
\text { exercise progression }\end{array}$ & Doses: Training load/intensity \\
\hline & $7 b$ & $\begin{array}{l}\text { Detailed description of how the exercise programme was } \\
\text { progressed }\end{array}$ & Doses: Training load/intensity \\
\hline & 8 & Detailed description of each exercise to enable replication & Additional file 2: Table S2 \\
\hline & 9 & Detailed description of any home programme component & Programme adherence \\
\hline & 10 & Describe whether there are any non-exercise components & Exercise programme rationale \\
\hline & 11 & $\begin{array}{l}\text { Describe the type and number of adverse events that occur } \\
\text { during exercise }\end{array}$ & Tally sheet/daily control (Additional file 3: Table S3) \\
\hline WHERE: location & 12 & Describe the setting in which the exercises are performed & Exercise programme characteristics \\
\hline $\begin{array}{l}\text { WHEN, HOW MUCH: } \\
\text { dosage }\end{array}$ & 13 & Detailed description of the exercise intervention & Weekly volume \\
\hline \multirow[t]{3}{*}{ TAILORING: what, how } & $14 a$ & $\begin{array}{l}\text { Describe whether the exercises are generic (one size fits all) or } \\
\text { tailored }\end{array}$ & Sessions structure and exercises \\
\hline & $14 b$ & Detailed description of how exercises are tailored to the individual & Sessions structure and exercises \\
\hline & 15 & Describe the decision rule for determining the starting level & Sessions structure and exercises \\
\hline \multirow[t]{2}{*}{$\begin{array}{l}\text { HOW WELL: planned, } \\
\text { actual }\end{array}$} & $16 a$ & Describe how adherence or fidelity is assessed/measured & $\begin{array}{l}\text { Programme adherence } \\
\text { Tally sheet/daily control (Additional file 3: Table S3) }\end{array}$ \\
\hline & $16 b$ & $\begin{array}{l}\text { Describe the extent to which the intervention was delivered as } \\
\text { planned }\end{array}$ & Tally sheet/daily control (Additional file 3: Table S3) \\
\hline
\end{tabular}




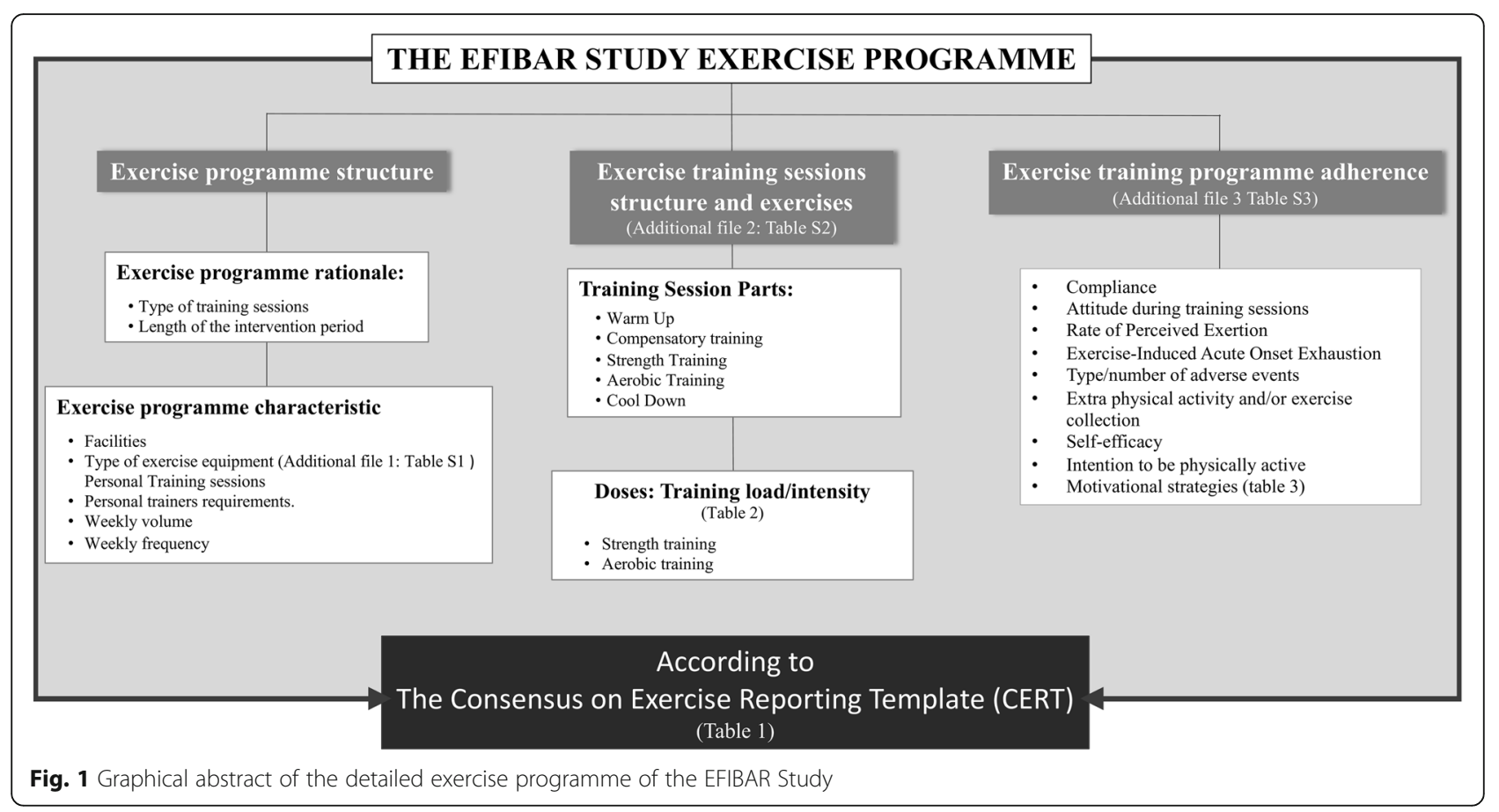

aerobic training generated a greater effect in reducing body fat compared to aerobic prior to strength training [27]. However, further research is still needed to know which order (i.e., strength prior to aerobic or vice versa) produces greater benefits in BS patients.

The length of the intervention period is 16 weeks based on a previous large scale RCT [28], where substantial physiological adaptations occurred within the first 3 to 6 months of exercise. We also considered the increased logistical and participation burdens, which indeed might lead to poorer adherence, as well as the cost of running a highly controlled laboratory-based study for a longer period. In addition to the exercise programme, all participants (both control and exercise groups) will undergo routine visits to the clinic during medical discharge and also for follow-up visits (months 1, 3, 6, 9 and 12). In these examinations, lifestyle-specific instructions (Item 10), including dietary and physical activity recommendations, will be provided as indicated in the perioperative clinical practice guidelines by the American Association of Clinical Endocrinologists, The Obesity Society, and American Society for Metabolic \& Bariatric Surgery [29].

\section{Exercise programme characteristics}

The exercise programme will be carried out in a fitness room at the University of Almería $\left(400 \mathrm{~m}^{2}\right)$, which is equipped with aerobic and strength training areas. The mean temperature registered in Almería from www. aemet.es averaged $19^{\circ}$ during 2018. The training programme will be free for participants (Item 12). The detailed description of the exercise equipment (Item 1) used in the training programme is shown in Additional file 1: Table S1. Two Personal Trainers (PT) with BSc degrees in Sport Sciences, and at least 2 years of experience as personal trainers, will be in charge of all sessions (Item 2). The PTs will receive a written standardised programme and weekly instruction by the study group training coordinators. Finally, the exercise programme will be developed individually (i.e., personal training sessions) (Item 3) and always supervised with a ratio of one-to-one (1,1, personal trainer, participant) (Item 4), unless participants report schedule unavailability; if this is the case, some sessions will be permitted to include two patients per PT.

\section{Weekly volume}

The prescription of the exercise programme will try to meet the recommendations proposed by current guidelines for aerobic and strength training in healthy adult populations [29]. The total exercise training volume will be $180 \mathrm{~min} /$ week. The doses will be composed of 48 sessions $(1 \mathrm{~h} /$ session) over 16 weeks ( 3 times/week). A full description of the training periodization (Item 13) is shown in Table 2.

It is estimated that $150 \mathrm{~min} /$ week of moderate intensity, i.e., $\approx 3-5.9$ metabolic equivalent (MET, $1 \mathrm{MET}=3.5 \mathrm{ml}$ $\mathrm{O}_{2} / \mathrm{kg} / \mathrm{min}$ ) aerobic physical activity is equivalent to 1000 $\mathrm{kcal} /$ week; this is associated with lower rates of cardiovascular disease and premature mortality in healthy adults [29]. Moreover, an energy expenditure of $1000 \mathrm{kcal} /$ week can also be achieved with $\approx 75 \mathrm{~min} /$ week of vigorous 
intensity ( $\geq 6$ METs) aerobic exercise. Thus, the proposed weekly volume of $180 \mathrm{~min} /$ week of concurrent training is likely to elicit $\approx 1200 \mathrm{kcal} /$ week. PTs will continuously monitor heart rate using a Polar Electro V-800 (Kempele, Finland) in all sessions of the intervention programme.

\section{Weekly frequency}

Although physical activity recommendations suggest undertaking physical activity most or preferably all days of the week, there was concern that exercising more than 3 days per week would be an excessive burden and might have undesirable effects on participants' adherence and motivation to complete the exercise intervention programme. Studies on exercise frequency show little differences for 3 or more days per week provided the weekly dose of exercise is attained [29]. Participants in the EFIBAR study will perform the exercise programme 3 days per week (preferably on Mondays, Wednesdays and Fridays). If the participant misses one training session, the participant will be rescheduled for a different day of the week to recover the session, provided the necessary between-session resting time is achieved (a minimum of $24 \mathrm{~h}$ ). A previous study of morbidly obese women found that three sessions per week improved cardiac parameters, such as variability and heart rate kinetic [30]. At the end of each training week, the PTs will remind participants of their appointments for the following week's training sessions to increase attendance.

\section{Sessions structure and exercises}

The main structure of the physical exercise sessions will be: 1) warm up; 2) compensatory training; 3) strength training; 4) aerobic training; and 5) cool down. A detailed description of the exercises used in the EFIBAR RCT (Item 8) is shown in Additional file 2: Table S2. Briefly, the warm up comprises $5 \mathrm{~min}$ of low intensity aerobic activity (treadmill) at 50 to $65 \%$ of heart rate reserve (HRR). Compensatory training includes core stability and stabiliser muscle exercises prior to strength training. Strength training will comprise a whole body exercise routine involving major upper and lower body muscle groups progressed through three phases after a familiarisation phase. Then, aerobic training will be conducted on a treadmill. Finally, participants will perform a cool down including static and dynamic stretching exercises.

In compensatory training, from week 3 until the end of the programme, participants will perform nine core stability and stabiliser muscle exercises, in order to minimise risk of injury and, hopefully, increase adherence (page 30 to 59 of Additional file 2: Table S2). All exercises will be modified such that they can be performed at three levels of difficulty: basic (Level A), intermediate (Level B) and advanced (Level C). All participants will begin at the basic level (Item 15). These exercises will be carefully progressed in level of difficulty (Item $7 a$ and $7 b$ ). In general, Level A will be carried out from week 3, Level B from week 5, and Level C from week 11. A certain degree of individualization will also be permitted, as each participant will progress through the different exercise levels (i.e., A, B or C) according to their individual adaptation; when the participant successfully complete all sets/repetitions of the prescribed exercises in a level (see Additional files 2 and 3) over more than three consecutive sessions (i.e., over 1 week), they will be able to progress to the next level. Our PTs will modify all exercises based on the participant's response to the exercise (Item 14a and 14b). The progression and order has been carefully structured, as presented in Additional file 2: Table S2. The proposed exercises allow for good activation of the central area of the body (e.g., abdominal area) with low pressure in the spinal structures (e.g., bird dog, side plank or modified crunch), as recommended in previous studies [31].

For strength training, the exercises will progress in intensity based on the participant's response to the exercise during the four main phases (Item $7 \mathrm{a}$ and 7b): familiarisation (weeks 1 to 4), phase 1 (weeks 5 to 8), phase 2 (weeks 9 to 10) and phase 3 (weeks 11 to 16). Participants will go through a familiarisation period during the first 4 weeks of the exercise program before including external loads. Since participants may present some movement limitations due to discomfort during the first weeks after surgery [32], we will prescribe exercises that allow participants to learn movement patterns (from week 1) and weight-bearing and strength training with elastic bands (from week 3) of the different main movements (e.g., squat, horizontal pull, vertical push); this will provide participants with the appropriate technique for the main exercises. In this phase, participants will learn seven movement pattern exercises, which constitute the basis of movement, such as diaphragmatic or abdominal breathing, dissociation and mobility of the hip or stabilization of the shoulders or the wrist. These seven exercises can be performed whenever the PT feels they are necessary over the 16 weeks in order to remind the patients of the basic movement patterns. During the first 4 weeks, participants will perform two sets of 5 to 7 repetitions of each movement pattern exercise. The resting time between series of these exercises will be adjusted to the patients' perceptions. Moreover, they will be asked to perform the exercises at progressively increasing speeds. It will be recommended that they 
do not perform trunk and hip flexion exercises until week 2 or 3 to avoid discomfort in the area of the surgical intervention (see the example of the hip flexion exercises 2 and 6 of movement pattern exercises in Additional file 2: Table S2). Likewise, in order to minimise trunk flexion during the first 2 to 3 weeks, we will avoid upward facing positions by proposing foot variants (see exercises 1 and 2 of the movement patterns exercises). Given the participants' degree of obesity, the order of the exercises was carefully designed to improve the flow of the sessions (i.e., reducing the number of transitions from lying to standing positions). Thus, the seven proposed exercises will be performed in the order detailed in Additional file 2: Table S2.

From phase 1 (week 5) onwards, all participants will perform exercises with external loads, including six main exercises performed in the following order: 1) squat; 2) seated lat pull-down; 3) bench press; 4) seated low row; 5) push press with dumbbells; and 6) deadlift. The training progression from one exercise to another will be done vertically (e.g., exercise 1 - rest - exercise 2). A detailed description of each exercise is presented in Additional file 2: Table S2, according to previous training recommendations [4] (Item 14a and 14b). Participants will be instructed to exercise through the full range of motion and to avoid the Valsalva manoeuvre. Finally, it should be noted that previous studies used similar strength training modalities in BS populations, such as stackweight equipment, free weights, body weight or resistive bands, in order to maintain high levels of enjoyment, engagement, and most importantly, adherence [33].

The aerobic training part will be conducted on a treadmill after strength training. Previous findings recommend that obese individuals can briskly walk or even run, provided they follow conservative transitions and progression, schedule rest days and heed onset of pain symptoms [34]. For progression to running, intensity or mileage increases should be slow and consistent to prevent musculoskeletal injury. Moreover, a study by Vincent et al. indicated that patients who have undergone bariatric surgery and are now lean can also run, but special foci, such as hydration and energy replacement, must be considered. All these considerations will be taken into account in the EFIBAR RCT.

Finally, participants will perform a cool down, including $5 \mathrm{~min}$ of static and dynamic stretching exercises in order to promote training adherence.

\section{Doses: training load/intensity}

Several public health institutions clearly indicate that moderate intensity physical activity is beneficial for health in deconditioned persons [23, 35], yet additional benefits have been observed for vigorous compared to moderate intensity exercise [29]. An intensity of at least $60 \%$ HRR is sufficient to produce clinically significant physiological adaptations in sedentary individuals $[23,29,36]$. We are aware that our participants might not be immediately capable of exercising at their required volume and intensity dose; therefore, there will be a gradual progression to the assigned exercise dose. The training loads in the EFIBAR RCT will range from $\approx 50$ to $75 \%$ of one repetition maximum (1 RM) for strength training, and from 65 to 85\% HRR for aerobic training. Moreover, the Borg Rating of Perceived Exertion (RPE) scale will also be used to monitor the intensity of aerobic training [36] (intensity values from 6 to 9 , where the scale is 1-10) and the OMNI-resistance scale (intensity values from 5 to 8 , where the scale is $1-10$ ) will be used for strength training [37].

Strength training An intensity equivalent to $40-50 \%$ of 1 RM may be beneficial for improving muscle strength in sedentary persons beginning a strength training programme, whereas $60-70 \%$ of $1 \mathrm{RM}$ is recommended for novice to intermediate exercisers to improve strength [24]. The main strength training phase (i.e., from weeks 5 to 16$)$ will have an intensity progressing from 24 to 10 $\mathrm{RM}(\approx 50$ to $75 \%$ of $1 \mathrm{RM})$, quantified by perceived exertion $(\mathrm{CE})$ as in previous studies $[38,39]$ (i.e., the maximum number of repetitions which participants could perform with a given load). In week 5 , participants will perform one set of 12 repetitions of strength training, lifting a load with which the participant could perform a maximum of 24 repetitions, whereas participants will finish the programme (week 16) performing three sets of 6 repetitions using the load corresponding to 10 maximum repetitions. $\mathrm{CE}$ will be readjusted twice during the exercise programme: at the beginning of phase 1 (session 13) and during phase 3 (session 31).

As the load (i.e., \% RM) is not the only variable that determines strength training intensity, we will control other variables such as movement speed during both concentric and eccentric phases, recovery time and range of motion; therefore, we assume that different loads (i.e., $\approx 50 \%$ RM vs. $75 \%$ RM) will constitute different training intensities $[24,40]$. The cadence for the strength exercises is fixed at 1:2 (concentric: eccentric phases), performing the concentric phase with the maximum velocity possible [41]. On the other hand, it is well known that different configurations of the strength training stimulus can elicit different physiological responses (e.g., muscle damage, metabolic stress) [42]. Taking into account that it is not known which kind of strength training stimulus is best for this population, we will vary the type of stimuli across the three different strength training phases. From 
weeks 5 to 8 , we will conduct strength training based on metabolic stress (i.e., <load and > volume), from weeks 9 to 11 it will be based on metabolic stress/ mechanical tension and muscle damage, and from week 12 to the end of the intervention we will conduct a programme based on mechanical tension and muscle damage stress (i.e., >load and $<$ volume), following previous strength training recommendations [40, 42]. For strength training with elastic bands (weeks 3 and 4), the intensity will be controlled by the Thera-Band RPE scale (range 2 to 6 , easy to somewhat hard) [43]. Rest periods between sets were intentionally minimised to 30 to $60 \mathrm{~s}$ to achieve the proposed intensity [44].

Aerobic training Aerobic training intensity will be controlled based on heart rate reserve (HRR). HRR will be calculated from the maximum real heart rate (HRmax) achieved during the maximum treadmill test, which will be performed at baseline as part of the pre-surgery evaluations. The Karvonen formula ([(maximum heart rate - resting heart rate) $\mathrm{x} \%$ training sensitive zone] + resting heart rate) will be used to calculate the individual exercise intensity. Resting heart rate will be taken from the heart rate variability (HRV) assessment included in the pre-surgery evaluations, as this test requires $10 \mathrm{~min}$ of sitting rest. Five training zones will be used: Zone $1<55-69 \%$; Zone 2: 70-79\%; Zone 3: 8084\%; Zone 4: 85-89\%; and Zone 5: >90\%. Heart rate monitors will be programmed according to the percentage of the participants individual HRRmax. In order to adjust the individual exercise intensity throughout the programme, the HRR will be estimated using the Karvonen formula in weeks 12 and 36. In addition, prior to the beginning of each exercise session, participants will wear a Polar heart rate monitor (V-800) and rest in a seated position for $5 \mathrm{~min}$, after which time their heart rate will be recorded to ensure that they are not beginning with a heart rate in high training zones [44].

Participants will start with a dose of $15 \mathrm{~min} / \mathrm{session}$ of aerobic training with $\leq 65 \%$ HRR during weeks 1 to 4 (familiarisation phase). From this point onwards, they will start a gradual increase in exercise volume and intensity. The volume will increase to $20 \mathrm{~min} /$ session from 65 to $70 \%$ of HRR during weeks 5 to 8 (phase 1). The volume in phase 2 will be maintained $(20 \mathrm{~min} / \mathrm{session})$, but with an increase of $5 \%$ in intensity (i.e., until $75 \%$ of HRR). The first week of phase 3 will have the same volume as phase $2(20$ $\mathrm{min} / \mathrm{session}$ ), but from weeks 12 to 16 it will be increased by $5 \mathrm{~min}$ (i.e., until $25 \mathrm{~min} / \mathrm{session}$ ) with intensity from 75 to $85 \%$ of HRR. We will estimate intensity (\% of HRR) by monitoring all sessions with Polar heart rate monitors. Moreover, the CR-10 Borg scale $[45,46]$ (values ranging from 0 to 10 ) will be used to evaluate perceived exertion during the aerobic part (range between 7 to 9 corresponding to 75 to $85 \%$ of HRR) and after that in order to control the final intensity in this part.

Whenever the aerobic target intensity (\% of HRR) is not reached by means of speed increase (without running), the inclination of the treadmill will be increased. A previous study conducted in moderately obese adults (mean BMI $33.4 \mathrm{~kg} / \mathrm{m}^{2}$ ) demonstrated that walking at slower speeds and on moderate inclines lowered the net muscle moments of the joints of the lower extremities [47]. Furthermore, following recommendations from this study, in the EFIBAR $\mathrm{RCT}$, the treadmill inclination will be increased by a maximum of $1^{\circ}$ every 2 weeks, and the final inclination will not exceed $6^{\circ}$ to minimise the risk of the onset of chronic tibia pain [48].

\section{Programme adherence}

Adherence to the exercise programme will be measured throughout the whole intervention period using a comprehensive tally sheet to be completed daily by the PT during and after each training session. The detailed tally sheet is shown in Additional file 3: Table S3. It describes measurement and reporting of adherence to the exercise programme (Item 5), the type and number of adverse events that occur during exercise (Item 11), the adherence and fidelity to the programme (Item 16a and 16b). Moreover, extra physical activity and/or exercise performed (Item 9) will be register as it has been recommended [46, 47]. Briefly, training attendance will be defined as the number of sessions attended divided the number of sessions prescribed $(n=48)$. Performing at least $80 \%$ of all planned training sessions will be considered a successful attendance rate. Additionally, self-efficacy and intention to be physically active will be measured at baseline (pre-surgery), postintervention (4 months after surgery) and at 1-year followup examinations, due to their associations with adherence to the exercise programmes [48]. For self-efficacy, we will use a modification of the McAuley's Exercise Self Efficacy Scale [48], whereas for measuring the intention to be physically active, we will adopt the question used by GonzálezCutre et al. [49]. During each training session, other variables related to adherence to the exercise programme will be collected, such as punctuality, extra physical activity, compliant attitude, and the Rate of Perceived Exertion for aerobic and strength training (Borg Scale RPE: 0-10 and ONMI resistance scale: $0-10$, respectively). Heart rate (using a Polar V800 pulsometer) will be registered immediately after each part of the training session. Mood through Feeling Scale, Exercise-Induced Acute Onset Exhaustion by HPHEE scale [50] and the Rate of Perceived Exertion by Borg Scale (RPE 0-10) will be also measured before and after training sessions. All of these variables are presented in Additional file 3: Table S3. Another adherence strategy 
designed to maintain motivation throughout the intervention consists of WhatsApp motivational messages and videos (Item 6), which will be sent to programme participants; participants will receive WhatsApp messages every Friday and videos every month. The 16 motivational messages and four videos are shown in Table 2.

\section{Discussion}

The EFIBAR RCT aims to investigate the effect of a 16week supervised exercise programme on weight loss, body composition, cardiometabolic risk, physical fitness and quality of life in morbidly obese patients following BS. Physical exercise in the clinical setting has increased its presence due to the numerous benefits reported in a wide range of patients [51]. However, the description of exercise-based interventions in clinical trials has largely been poor [52]. CERT was recently developed as a tool to enhance replicability of exercise interventions.

To the best of our knowledge, this is the first evidence-based description of an exercise programme in morbid obese individuals based on CERT methodology. However, it should be noted that previous results in BS and obese patients have been considered and incorporated into the design of the present programme. For instance, Huck et al. [53] found that supervised strength training using free weights, body weight, or resistance bands safely improved muscular strength and physical functioning, increasing the patient's capacity to perform daily activities after BS. In this study, each training session comprised 8-10 exercises targeting all major muscle groups, although the specific exercises performed were not reported. Castello-Simoes et al. [30] implemented diaphragmatic breathing to prepare the body for the subsequent activity. Colato et al. [54] employed a concurrent training programme using stack-weight equipment and concluded that an exercise training programme in the first 4 months is effective for improving body composition in overweight and obese patients (BMI $25-39.9 \mathrm{~kg} / \mathrm{m}^{2}$ ). Consequently, in the current study, participants will start to train as soon as possible (1-2 weeks after BS), although the first increase in strength/aerobic intensity and volume will not occur until 1 month after surgery. Thus, we have incorporated previous knowledge into the design of this evidencebased intervention.

It should be recognised that some criteria are derived from previous studies carried out in non-morbidly obese (or BS) individuals, since the evidence in BS individuals is scarce. For example, the concurrent training order (i.e., strength before aerobic training) was established following studies in obese (but not BS) populations, in which this order was the most beneficial for improving body composition, physical fitness and other health biomarkers [54]. The structure of the exercise programme is the same as that of Colato et al. [54] in which 14 overweight and obese adults carried out $60 \mathrm{~min}$ training sessions, each including three parts: 1) warm-up exercises; 2) concurrent training; and 3) stretching. In our study, even while following the same structure, the intensity and training volume will adjust and progressively increase throughout the 16 weeks. In addition, we are aware that starting the training program 7-14 days after BS can be quite early for this patients. However, participants will go through a familiarisation period during the first 4 weeks, which only includes walking at low-moderate intensity and some exercises aimed at learning movement patterns, as well as exercises with a low speed and a high body control for a better body posture. Also, participants will follow a gradual progression to reach the assigned exercise dose. Additionally, our detailed tally sheet (Additional file 3: Table S3) will register the ExerciseInduced Acute Onset Exhaustion by HPHEE scale, the Rate of Perceived Exertion by Borg Scale (RPE 0-10) before and after each training session, as well as possible adverse events that may occur during exercise.

In this evidence-based exercise programme for BS patients, the exercise techniques and their rationale are described in detail, together with advice to carry them out appropriately. This ensures understanding by different health professionals from multidisciplinary teams, although the exercise professionals (i.e., well-qualified PTs) should play a relevant role in its implementation [55]. Exercise progressions have been designed such that the participants (and their PTs) can choose to increase or reduce the exercise difficulty (i.e., Level A, B or C) to allow all BS patients to undertake the programme. The main strength exercises are common and easy to perform, in order to be replicable in other contexts (e.g., clinical settings, fitness centers, research laboratories). Moreover, this programme includes a large number of body weight exercises that can be performed without any equipment, increasing its applicability in other studies or contexts.

Adherence to an exercise programme is fundamental to understanding the clinical efficacy of exercise-based interventions [56]. Consequently, we have carefully revised the BS literature with special attention to adherence strategies for exercise programmes. Wiklund and colleagues [57] conducted semi-structured interviews with 18 patients awaiting bariatric surgery. They reported that these patients tend to be uncomfortable appearing in public wearing exercise clothing and that exercising with someone at the same level of health/fitness increased motivation [57]. Therefore, to avoids patients' discomfort and to increase safety levels, we selected individual sessions (i.e., ratio 1:1), in which the PTs will monitor the level of adherence using a comprehensive tally sheet (Additional file 3: Table S3) during and after 


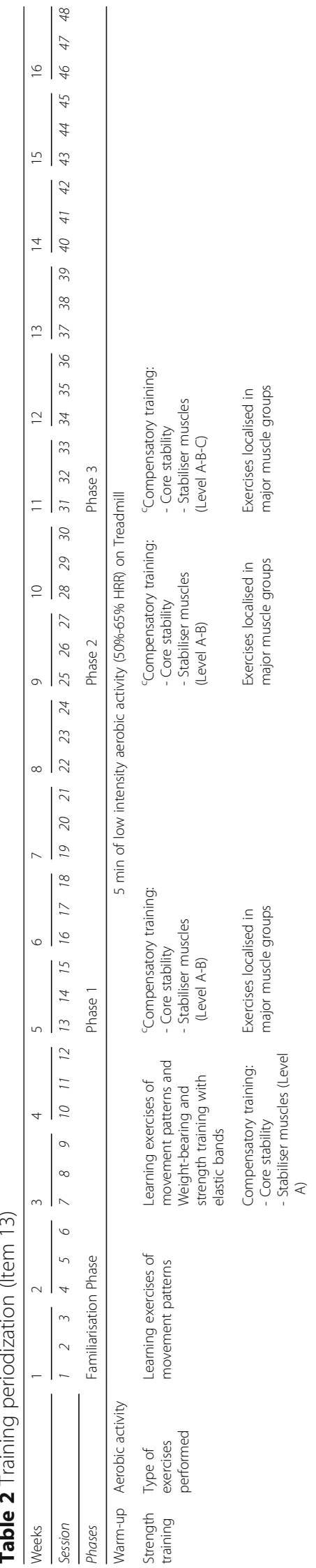

$m \circ \stackrel{+}{\circ} \stackrel{\infty}{\sim}$

$m \bullet \odot+\infty \frac{\overline{\bar{\varepsilon}}}{\sim}$

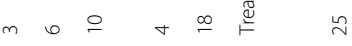

$m 0 \simeq 0 \simeq$

$m 0 \simeq 0 \simeq \frac{\overline{\bar{\varepsilon}}}{n}$

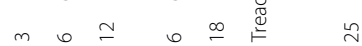

$m \infty \pm 0 \pi$

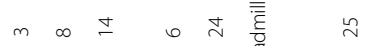

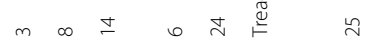

$m \infty \simeq \infty \underset{\sim}{4}$

$m \infty \simeq \infty$ オ

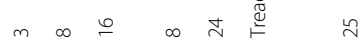

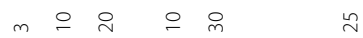

$m \simeq 0 \subseteq 0 \bar{m}$

$m \simeq 2 \bigcirc 0$ 兽山

$m \subseteq i \simeq \infty$

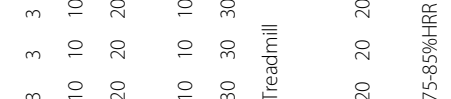

$m \simeq \dot{n} \simeq \infty$

$m \simeq \dot{\sim} \simeq \infty_{m}^{\overline{\bar{\varepsilon}}}$ \&

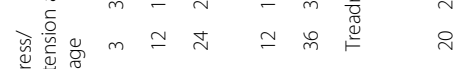

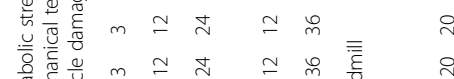

एँ

$-\simeq \pm \simeq \simeq$

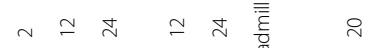

$\sim \simeq \dot{4} \simeq \underset{d}{ }$

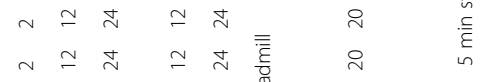

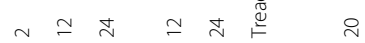

- $\simeq \underset{4}{ } \simeq \simeq$

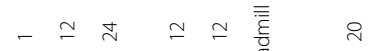

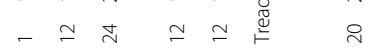

- $\simeq \underset{4}{ } \simeq \simeq$

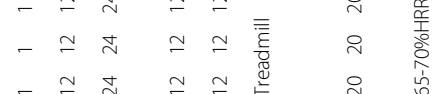

产

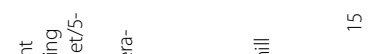


each training session. Furthermore, it is expected that the motivational WhatsApp and video messages will maintain patients' motivation and confidence throughout the intervention. Accordingly, previous literature has demonstrated that health club members whose training is directed by well-qualified PTs administering evidence-based training regimens achieve significantly greater improvements in lean body mass and other dimensions of fitness than members who direct their own training. [55]. Thus, the strategies have been designed to maximise adherence and efficacy of the EFIBAR exercise programme.

Knowing that some of the exercises had not been previously carried out in the literature, the EFIBAR RCT included a 2-week pilot programme before start recruiting participants, during which three patients with severe obesity (two women and one man) participated. These patients had different conditions, permitting observation of responses to the exercise programme from different points of view (i.e., a patient who did not expect surgery, a patient waiting for surgery and a patient who had undergone surgery 1 week earlier). The three patients tested the exercise progression for 2 weeks. Accordingly, some of the initially prescribed exercises were modified. For instance, some of the proposed progressions were changed, and other proposed exercises were simply removed (e.g., due to patients' discomfort). Finally, others have described that it is difficult to assess the appropriate type and amount of physical activity and/or exercise for BS patients, and it is unclear if preoperative exercise has the same effect as postoperative exercise [12]. Thus, it should be noted that the EFIBAR RCT exercise programme was carefully designed to be carried out after surgery, although we believe that it could also be conducted as a preoperative exercise programme.

Altogether, this report presents an evidence-based exercise intervention, following the CERT reporting guidelines, and a comprehensive rationale underlying each step conducted during its design. Consequently, this evidence-based report can not only be used for exercise prescription for morbidly obese individuals, but can also inform the use of the CERT guidelines for the comprehensive and transparent reporting of exercise interventions in BS patients and for patients with other diseases.

The present study details the exercise programme of the EFIBAR RCT, which may serve: 1) exercise professionals who would like to implement an evidence-based exercise programme for BS patients, and 2) as an example of the application of the CERT criteria. To the best of our knowledge, this is the first report of an exercise protocol based on CERT methodology in BS patients.
The results of the EFIBAR study will be disseminated through a strategic dissemination plan. The strategy will use different channels to reach a large number of stakeholder groups and individuals, at the local, national and international level; this will include dissemination in academic media (ie, peer-reviewed journal articles, national and international conference presentations), social media (ie, Facebook, Twitter), print media (ie, newspaper), broadcast media (ie, radio, television), the internet (ie, links to study reports on the University of Almería website), electronic and postal mail (ie, posting of study findings to participants and stakeholders) and community/stakeholder engagement activities (ie, community forums, stakeholder meetings).

\section{Additional files}

Additional file 1: Table S1. Exercise equipment for The EFIBAR study. (DOCX 2428 kb)

Additional file 2: Table S2. Detailed description of The EFIBAR exercises Training Programme. (DOCX $18742 \mathrm{~kb}$ )

Additional file 3: Table S3. Detailed tally sheet used to assess adherence to exercise programme (Item 5), type and number of adverse events that occur during exercise (Item 11), and fidelity (Item 16a and 16b). (DOCX 29 kb)

\section{Abbreviations}

BS: Bariatric surgery; CERT: Consensus on Exercise Reporting Template; HRmax: Maximum real heart rate; HRR: Heart rate reserve; HRV: Heart rate variability; MET: Metabolic equivalent; PT: Personal Trainers; RCT: Randomised controlled trials; RM: Repetition maximum; RPE: Rating of Perceived Exertion

\section{Acknowledgements}

The authors acknowledge the help of the participants that are taking part in the study. Moreover, authors appreciate the help to the hospitals, as well as the sports center of the University of Almería, who helped with the development of the project.

\section{Authors' contributions}

EVG, YBR, ASM and EGA were involved in the conception and design of the study, drafted the protocol, supervised the revisions and approved the final manuscript.

MRP, ACR, AHM, JMG, MJT, MFM were involved in the conception and design of this study, revised the draft critically for intellectual content and approved the final revised manuscript. All the authors have revised and approved the final version of the manuscript.

\section{Funding}

This work was supported by Ministerio de Economía y Competitividad (MINECO), Plan Nacional de I + D + i call RETOS 2016, reference DEP2016-74926-R. These funding sources approved this study; however, they had no role in the design of this study and will not have any role during its execution, analyses, interpretation of the data, writing the manuscript, or decision to submit results.

\section{Availability of data and materials}

The clinical datasets will be available according to the terms established in the Clinicaltrials.gov NCT03497546, i.e., once the study ends and the main results are published, contacting the responsible party (artero@ual.es).

\section{Ethics approval and consent to participate}

The design of the study, assessment and intervention protocols, as well as informed consents, were reviewed and approved by the Ethics Committee of the Torrecárdenas University Hospital prior to the start of the recruitment process (case $N^{\circ}$ 76/2016). Each patient received a dossier with detailed 
information about the study design, general purpose, assessment protocols and interventions. The surgeon provided a comprehensive description of the project to each participant. Participants were not evaluated for inclusion before signing their approval for participation in the project.

\section{Consent for publication}

Approximately 1 month before the surgery, each participant is informed of the study design, general purpose, assessment protocols and interventions. All participants will receive detailed information about possible outcomes that could have an impact on their health. In this case, the medical personnel and researcher will inform participants and give advice. A written summary of the study results will be sent to participants by email. Participants in this study shall complete informed consent and shall receive a copy of results and publications from this work. In addition, all participants will receive a certificate of appreciation for participating in the study. Participants will not be involved in the design and conception of the study. In addition, written informed consent have been collected from all participants who appear in these photographs, giving us explicit permission to use their photographs for scientific / educational purposes.

\section{Competing interests}

The authors declare that they have no competing interests.

\section{Author details}

${ }^{1}$ Department of Physical and Sports Education, PROFITH "PROmoting FITness and Health through Physical Activity" Research Group, Sport and Health University Research Institute (iMUDS), Faculty of Education and Sport Sciences, University of Granada, 52005 Melilla, Spain. ${ }^{2}$ Department of Education, Faculty of Education Sciences, University of Almería, Almería, Spain. ${ }^{3}$ SPORT Research Group (CTS-1024), CERNEP Research Center, University of Almería, Almería, Spain. ${ }^{4}$ Hospital Mediterráneo, Almería, Spain. ${ }^{5}$ Torrecárdenas University Hospital, Almería, Spain.

Received: 27 March 2019 Accepted: 19 July 2019

Published online: 05 September 2019

\section{References}

1. Ribaric G, Buchwald JN, McGlennon TW. Diabetes and weight in comparative studies of bariatric surgery vs conventional medical therapy: a systematic review and meta-analysis. Obes Surg. 2014;24:437-55.

2. Sjöström L, Lindroos A-K, Peltonen M, Torgerson J, Bouchard C, Carlsson B, et al. Lifestyle, diabetes, and cardiovascular risk factors 10 years after bariatric surgery. N Engl J Med. 2004;351:2683-93.

3. Courcoulas AP, Christian NJ, Belle SH, Berk PD, Flum DR, Garcia L, et al. Weight change and health outcomes at 3 years after bariatric surgery among individuals with severe obesity. JAMA. 2013;310:2416-25.

4. Magro DO, Geloneze B, Delfini R, Pareja BC, Callejas F, Pareja JC. Long-term weight regain after gastric bypass: a 5 -year prospective study. Obes Surg. 2008;18:648-51.

5. Steele T, Cuthbertson DJ, Wilding JPH. Impact of bariatric surgery on physical functioning in obese adults. Obes Rev. 2015;16:248-58.

6. Shah M, Simha V, Garg A. Review: long-term impact of bariatric surgery on body weight, comorbidities, and nutritional status. J Clin Endocrinol Metab. 2006;91:4223-31.

7. Bond DS, Vithiananthan S, Thomas JG, Trautvetter J, Unick JL, Jakicic JM, et al. Bari-active: a randomized controlled trial of a preoperative intervention to increase physical activity in bariatric surgery patients. Surg Obes Relat Dis. 2015;11:169-77.

8. Obesity: Identification, Assessment and Management of Overweight and Obesity in Children, Young People and Adults: Partial Update of CG43. Natl Inst Health Clin Excell Guid. London: National Institute for Health and Care Excellence; 2014

9. Josbeno DA, Jakicic JM, Hergenroeder A, Eid GM. Physical activity and physical function changes in obese individuals after gastric bypass surgery. Surg Obes Relat Dis. 2010;6:361-6.

10. Yale CE. Gastric surgery for morbid obesity. Complications and long-term weight control. Arch Surg. 1989;124:941-6.

11. Wittgrove AC, Clark GW. Laparoscopic gastric bypass, Roux-en-Y- 500 patients: technique and results, with 3-60 month follow-up. Obes Surg. 2000;10:233-9.

12. Pouwels S, Wit M, Teijink JAW, Nienhuijs SW. Aspects of exercise before or after bariatric surgery: a systematic review. Obes Facts. 2015;8:132-46.
13. Bellicha A, Ciangura C, Poitou C, Portero P, Oppert J-M. Effectiveness of exercise training after bariatric surgery-a systematic literature review and meta-analysis. Obes Rev. 2018;19:1544-56.

14. Ballor $\mathrm{DL}$, Katch VL, Becque MD, Marks CR. Resistance weight training during caloric restriction enhances lean body weight maintenance. Am J Clin Nutr. 1988;47:19-25.

15. Geliebter A, Maher MM, Gerace L, Gutin B, Heymsfield SB, Hashim SA. Effects of strength or aerobic training on body composition, resting metabolic rate, and peak oxygen consumption in obese dieting subjects. Am J Clin Nutr. 1997;66:557-63.

16. Montero D, Roberts CK, Vinet A. Effect of aerobic exercise training on arterial stiffness in obese populations : a systematic review and metaanalysis. Sports Med. 2014;44:833-43.

17. Coen PM, Goodpaster BH. A role for exercise after bariatric surgery? Diabetes Obes Metab. 2016;18:16-23.

18. Slade SC, Dionne CE, Underwood M, Buchbinder R. Consensus on Exercise Reporting Template (CERT): Explanation and Elaboration Statement. Br J Sports Med. 2016;50:1428-37.

19. Slade SC, Keating $\mathrm{J}$. Exercise prescription: a case for standardised reporting. Br J Sports Med. 2012;46:1110-3.

20. Soriano-Maldonado A, Villa-González E, Ferrer-Márquez M, Artero EG. Replicability of exercise programs following bariatric surgery. Atherosclerosis. 2018;278:330-1.

21. Mechanick JI, Youdim A, Jones DB, Garvey WT, Hurley DL, McMahon MM, et al. Clinical practice guidelines for the perioperative nutritional, metabolic, and nonsurgical support of the bariatric surgery patient--2013 update: cosponsored by American Association of Clinical Endocrinologists, The Obesity Society, and American Society f. Obesity. 2013;21(Suppl 1):S1-27.

22. Colton T, Armitage P. Encyclopedia of biostatistics. New York: Wiley; 2005.

23. WHO. WHO, Global recommendations on physical activity for health. Switzerland; 2010.

24. American College of Sports Medicine. American College of Sports Medicine position stand. Progression models in resistance training for healthy adults. Med Sci Sports Exerc. 2014;41:687-708.

25. Chlif M, Chaouachi A, Ahmaidi S. Effect of aerobic exercise training on ventilatory efficiency and respiratory drive in obese subjects. Respir Care. 2017;62:936-46.

26. Jones TW, Howatson G, Russell M, French DN. Effects of strength and endurance exercise order on endocrine responses to concurrent training. Eur J Sport Sci. 2017;17:326-34.

27. Sheikholeslami-Vatani D, Siahkouhian M, Hakimi M. The effect of concurrent training order on hormonal responses and body composition in obese men. Sci Sport. 2015;30:335-41. https://doi. org/10.1016/j.scispo.2015.06.005.

28. Pedersen BK, Saltin B. Exercise as medicine-evidence for prescribing exercise as therapy in 26 different chronic diseases. Scand J Med Sci Sports. 2015;25:1-72.

29. Garber CE, Blissmer B, Deschenes MR, Franklin BA, Lamonte MJ, Lee IM, et al. American College of Sports Medicine position stand. Quantity and quality of exercise for developing and maintaining cardiorespiratory, musculoskeletal, and neuromotor fitness in apparently healthy adults: guidance for prescribing exercise. Br J Sports Med. 2011;43:1334-59.

30. Castello-Simoes V, Polaquini Simoes R, Beltrame T, Bassi D, Maria Catai A, Arena $R$, et al. Effects of aerobic exercise training on variability and heart rate kinetic during submaximal exercise after gastric bypass surgery--a randomized controlled trial. Disabil Rehabil. 2013;35:334-42.

31. McGill SM, Karpowicz A. Exercises for spine stabilization: motion/motor patterns, stability progressions, and clinical technique. Arch Phys Med Rehabil. 2009;90:118-26.

32. Coleman KJ, Caparosa SL, Nichols JF, Fujioka K, Koebnick C, McCloskey KN, et al. Understanding the capacity for exercise in post-bariatric patients. Obes Surg. 2017:27:51-8.

33. Huck CJ. Effects of supervised resistance training on fitness and functional strength in patients succeeding bariatric surgery. J strength Cond Res. 2015;29:589-95.

34. Vincent HK, Vincent KR. Considerations for initiating and progressing running programs in obese individuals. PM R. 2013;5:513-9.

35. Services D. of H and H. Physical Activity Guidelines for Americans. 2008. http:// www.health.gov/PAGuidelines/.

36. Coquart JB, Tourny-Chollet C, Lemaitre F, Lemaire C, Grosbois JM, Garcin M. Relevance of the measure of perceived exertion for the rehabilitation of obese patients. Ann Phys Rehabil Med. 2012;55:623-40. 
37. Lagally KM, Robertson RJ. Construct validity of the OMNI resistance exercise scale. J Strength Cond Res. 2006;20:252-6.

38. Ferrari R, Kruel LF, Cadore EL, Alberton CL, Izquierdo M, Conceicao M, et al. Efficiency of twice weekly concurrent training in trained elderly men. Exp Gerontol. 2013;48:1236-42.

39. Ferrari R, Fuchs SC, Kruel LF, Cadore EL, Alberton CL, Pinto RS, et al. Effects of different concurrent resistance and aerobic training frequencies on muscle power and muscle quality in trained elderly men: a randomized clinical trial. Aging Dis. 2016;7:697-704.

40. Schoenfeld BJ, Ratamess NA, Peterson MD, Contreras B, Sonmez GT, Alvar BA. Effects of different volume-equated resistance training loading strategies on muscular adaptations in well-trained men. J Strength Cond Res. 2014;28:2909-18.

41. Zapico AG, Benito PJ, Gonzalez-Gross M, Peinado AB, Morencos E, Romero $B$, et al. Nutrition and physical activity programs for obesity treatment (PRONAF study): methodological approach of the project. BMC Public Health. 2012;12:1100.

42. Schoenfeld BJ. The mechanisms of muscle hypertrophy and their application to resistance training. J Strength Cond Res. 2010;24:2857-72.

43. Colado JC, Garcia-Masso X, Triplett NT, Calatayud J, Flandez J, Behm D, et al. Construct and concurrent validation of a new resistance intensity scale for exercise with thera-band(R) elastic bands. Br J Sports Med. 2014;13:758-66.

44. Swift DL, Dover SE, Nevels TR, Solar CA, Brophy PM, Hall TR, et al. The intervention composed of aerobic training and non-exercise physical activity (I-CAN) study: Rationale, design and methods. Contemp Clin Trials. 2015;45(Pt B):435-42.

45. Zamuner AR, Moreno MA, Camargo TM, Graetz JP, Rebelo AC, Tamburus NY, et al. Assessment of subjective perceived exertion at the anaerobic threshold with the Borg CR-10 scale. Br J Sports Med. 2011;10:130-6.

46. Soriano-Maldonado A, Márquez MF, Artero EG. Pain and physical function following bariatric surgery. JAMA. 2016;316:770-1.

47. Artero EG, Ferrer-Márquez M, Soriano-Maldonado A. When will physical activity be routinely measured in the clinical setting? The case for bariatric surgery. Am J Hypertens. 2016;29:e1.

48. McAuley E. Self-efficacy and the maintenance of exercise participation in older adults. J Behav Med. 1993;16:103-13.

49. Gonzalez-Cutre D, Sicilia A, Beas-Jimenez M, Hagger MS. Broadening the trans-contextual model of motivation: a study with Spanish adolescents. Scand J Med Sci Sports. 2014;24:e306-19.

50. Hecimovich MD, Peiffer JJ, Harbaugh AG. Development and psychometric evaluation of a post exercise exhaustion scale utilising the Rasch measurement model. Psychol Sport Exerc. 2014;15:569-79.

51. Warburton DE, Nicol CW, Bredin SS. Health benefits of physical activity: the evidence. CMAJ. 2006;174:801-9.

52. Hoffmann TC, Erueti C, Glasziou PP. Poor description of nonpharmacological interventions: analysis of consecutive sample of randomised trials. BMJ. 2013;347:f3755.

53. Huck CJ. Effects of supervised resistance training on fitness and functional strength in patients succeeding bariatric surgery. J Strength Cond Res. 2015; 29:589-95.

54. Colato A, Abreu F, Medeiros N, Lemos L, Dorneles G, Ramis T, et al. Effects of concurrent training on inflammatory markers and expression of CD4, CD8, and HLA-DR in overweight and obese adults. J Exerc Sci Fit. 2014;12:55-61.

55. Muth ND, Vargo K, Bryant CX. The role of the fitness professional in the clinical setting. Curr Sports Med Rep. 2015;14:301-12.

56. Woodard CM, Berry MJ. Enhancing adherence to prescribed exercise: structured behavioral interventions in clinical exercise programs. J Cardiopulm Rehabil. 2001;21:201-9.

57. Wiklund $M$, Olsen MF, Willen C. Physical activity as viewed by adults with severe obesity, awaiting gastric bypass surgery. Physiother Res Int. 2011;16:179-86.

\section{Publisher's Note}

Springer Nature remains neutral with regard to jurisdictional claims in published maps and institutional affiliations.

\section{Ready to submit your research? Choose BMC and benefit from:}

- fast, convenient online submission

- thorough peer review by experienced researchers in your field

- rapid publication on acceptance

- support for research data, including large and complex data types

- gold Open Access which fosters wider collaboration and increased citations

- maximum visibility for your research: over $100 \mathrm{M}$ website views per year

At BMC, research is always in progress.

Learn more biomedcentral.com/submissions 